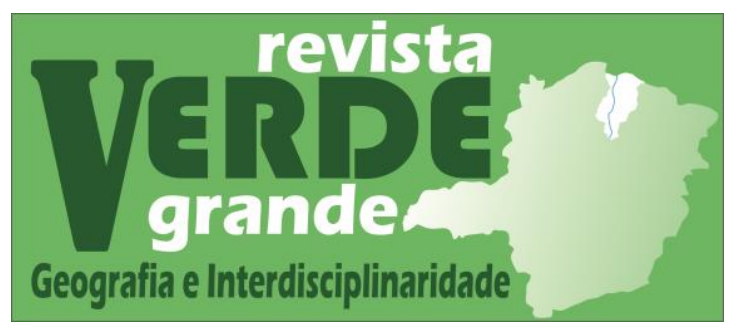

\title{
MEIO AMBIENTE, BOTÂNICA E SOLOS: UMA RELAÇÃO FUNCIONAL PARA AULAS DE BIOLOGIA E GEOGRAFIA.
}

\author{
Pedro Henrique Fonseca Veloso ${ }^{1}$ https://orcid.org/0000-0003-2802-1244 \\ Júlia Maria Viana Santana ${ }^{2}$ https://orcid.org/0000-0003-3306-4839 \\ Flávia Núbia Oliveira Miranda D’angelis ${ }^{3}$ https://orcid.org/0000-0002-1364-8880 \\ Ana Paula Venuto Moura ${ }^{4}$ https://orcid.org/0000-0002-3103-7063 \\ Guilherme Araújo Lacerda ${ }^{5}$ https://orcid.org/0000-0003-0878-6557
}

\footnotetext{
${ }^{1}$ Graduando em Ciências Biológicas Licenciatura pela Universidade Estadual de Montes Claros. E-mail: pedrofonsecambc@gmail.com

${ }^{2}$ Graduanda em Ciências Biológicas Licenciatura pela Universidade Estadual de Montes Claros. E-mail: juliasvs@gmail.com

${ }^{3}$ Professora supervisora do PIBID da Universidade Estadual de Montes Claros. E-mail: flavianom87@ gmail.com

${ }^{4}$ Coordenadora do PIBID Biologia da Universidade Estadual de Montes Claros. E-mail: apvenuto@yahoo.com.br

${ }^{5}$ Coordenador do PIBID Biologia da Universidade Estadual de Montes Claros. E-mail: guilherme.lacerda@ unimontes.br
}

\section{Resumo}

O ensino da biologia costuma ser regular de forma linear e em sala de aula, dificilmente existe a relação de ensino-aprendizagem fora da sala, em um espaço aberto onde a comunicação interpessoal se torna fluida e proveitosa, visando isso, as aulas práticas demonstrativas expositivas são uma excelente forma de trabalhar com diversos conteúdos, como é o caso da educação ambiental aplicada ao bioma da região, botânica onde o conteúdo constantemente é malvisto pela dificuldade de assimilação e a relação dos solos que faz o fechamento da absorção de água do ecossistema. O trabalho de campo auxilia na fixação das questões trabalhadas em sala de aula, estabelecendo uma relação de ensinoaprendizagem favorável ao aluno, que desfruta das atividades de uma forma mais dinâmica.

Palavras-chave: Educação Ambiental, Aula Prática, Ensino-aprendizagem.

\section{Sobre o Cerrado e a Botânica}

O Cerrado é a savana com maior biodiversidade do planeta, um bioma brasileiro que abriga uma grande biodiversidade vegetal de plantas vasculares nativas e endêmicas (Scariot, 2005; Maracahipes, 2011), de acordo com Kink e Machado (2005) o cerrado tem um longo histórico de desmatamento, superando os níveis de desmatamento da floresta Amazônica, enfatizando as recorrente de queimadas realizadas para ocorrência do brotamento de pastagens, esse processo de desmatamento causa mudanças, como o brotamento de gramíneas 
Meio Ambiente, botânica e solos: uma relação funcional para aulas de Biologia e Geografia

Pedro Henrique Fonseca Veloso; Júlia Maria Viana Santana; Flávia Núbia Oliveira Miranda D’angelis; Ana Paula Venuto Moura; Guilherme Araújo Lacerda

e mudando a forma estrutural da vegetação original, mesmo o cerrado sendo adaptado e resistente às queimadas.

A Botânica é uma das áreas que apresentam maior dificuldade de assimilação de conteúdos no processo de ensino-aprendizagem. A dificuldade maior dos professores e o desinteresse dos alunos quando o assunto é o estudo da anatomia vegetal soma-se à falta de aulas práticas e materiais didáticos que visem facilitar esse aprendizado. No entanto, quando abordadas de forma mais didática, a morfologia e a anatomia vegetal podem ser utilizadas como base fundamental para o entendimento do papel das plantas na manutenção dos serviços ecológicos, nos diferentes ecossistemas (Nascimento-Silva e De Paiva. 2017).

Podemos definir dendrocronologia como estudo do crescimento das árvores, onde se analisa os anéis de crescimento como finalidade para estimar a idade, épocas de chuva e seca além dos processos ambientais e regenerativos pelos quais a árvore passou.

E no bioma Cerrado é possível fazer uma análise mais detalhada, como as marcas e cicatrizes de fogo presentes nos anéis de crescimento, estudo denominado dendropirocronologia.

O objetivo do trabalho foi demonstrar os aspectos do cerrado no Parque Estadual da Lapa Grande localizado na cidade de Montes Claros no estado de Minas Gerais ( Mapa 1), visando como principal questão a educação ambiental, a conservação e análise da relação fogo e dendrocronologia, uma vez que o parque sofre queimadas frequentes em decorrência do período de seca que ocorre na região, e a caracterização do solo de acordo com a mata preservada, onde há visitação.

Mapa 1: localização do Parque Estadual da Lapa Grande na cidade de Montes Claros

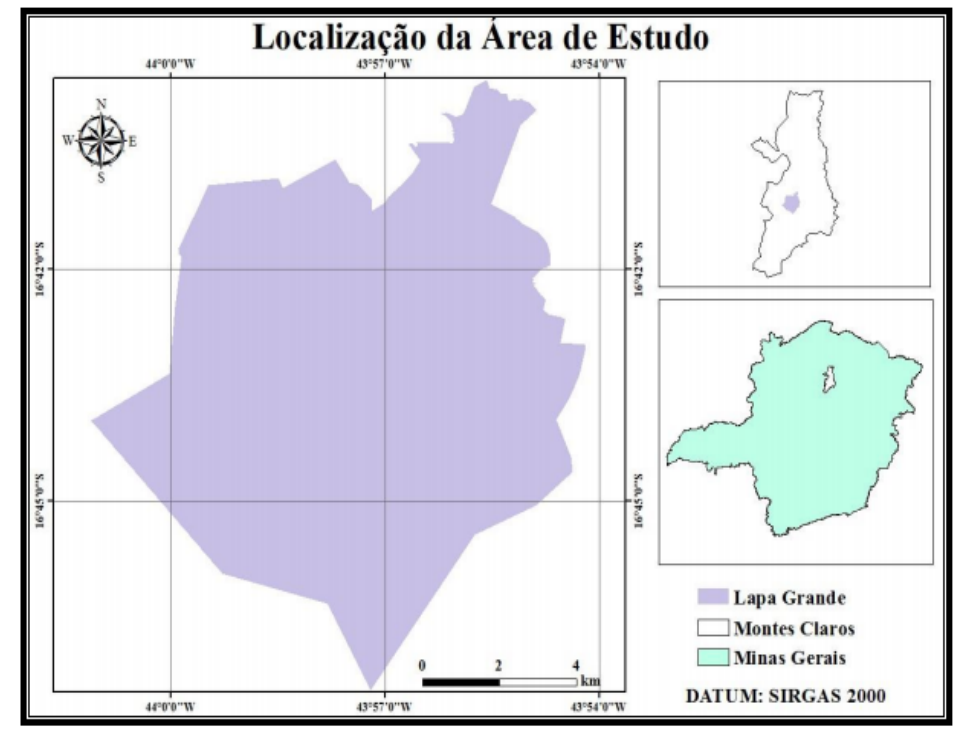

Fonte: MOREIRA et al, 2013, p. 1708 
Meio Ambiente, botânica e solos: uma relação funcional para aulas de Biologia e Geografia

Pedro Henrique Fonseca Veloso; Júlia Maria Viana Santana; Flávia Núbia Oliveira Miranda D’angelis; Ana Paula Venuto Moura; Guilherme Araújo Lacerda

\section{Em Sala, Em Prática}

Os materiais aplicados foram atividades sobre dendrocronologia, simulando uma árvore sobrevivente a uma queimada e análise dos seus anéis de crescimento em diferentes estações do ano (Figura 1), debate sobre a importância da conservação e análise de solo, realizado na Escola Estadual Antônio Canela.

A metodologia utilizada foi um trabalho de campo, uma aula expositiva demonstrativa realizada no Parque Estadual da Lapa Grande para fixação do conteúdo ministrado em sala de aula.

Figura 1: Alunos e discente na sala de aula observando os anéis de crescimento.

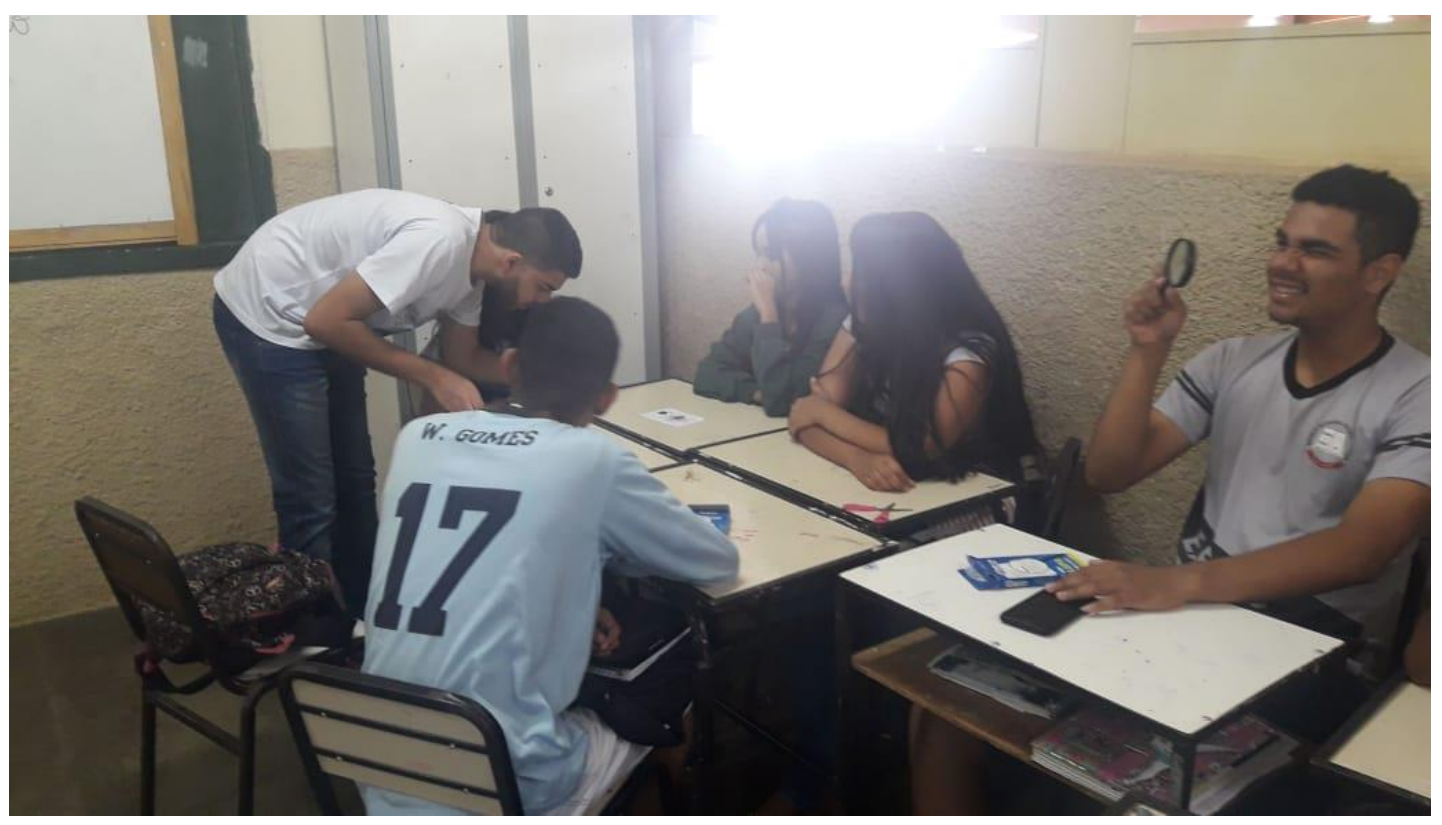

Fonte: Pedro Henrique Fonseca Veloso

\section{O Processo de Aprendizagem se Faz Presente}

De acordo com Carvalho (2017) é necessário a formação de um sujeito ecológico, formado a partir de psicologia social aplicada a educação ambiental, onde há a necessidade de levar essa problematização em todos o s níveis do ensino, com isso a autora enfatiza os processos socioculturais de preservação do meio ambiente.

A experiência de sair da sala de aula para um trabalho de campo é fundamental para o processo de ensino-aprendizagem onde o aluno se envolve com o meio, mostrando-se extremamente funcional e didático, com o auxílio dos discentes do curso de ciências biológicas licenciatura da Universidade Estadual de Montes Claros, a professora regente da 
Meio Ambiente, botânica e solos: uma relação funcional para aulas de Biologia e Geografia

Pedro Henrique Fonseca Veloso; Júlia Maria Viana Santana; Flávia Núbia Oliveira Miranda

D’angelis; Ana Paula Venuto Moura; Guilherme Araújo Lacerda

disciplina biologia e os guias do parque os alunos foram "desbravando" os arredores do parque onde foram debatidos diversos aspectos da vegetação preservada e a importância da sua conservação, como pode ser observado nas figuras 2,3 e 4 .

Figura 2. Procedimentos e orientações para fazer a trilha na mata

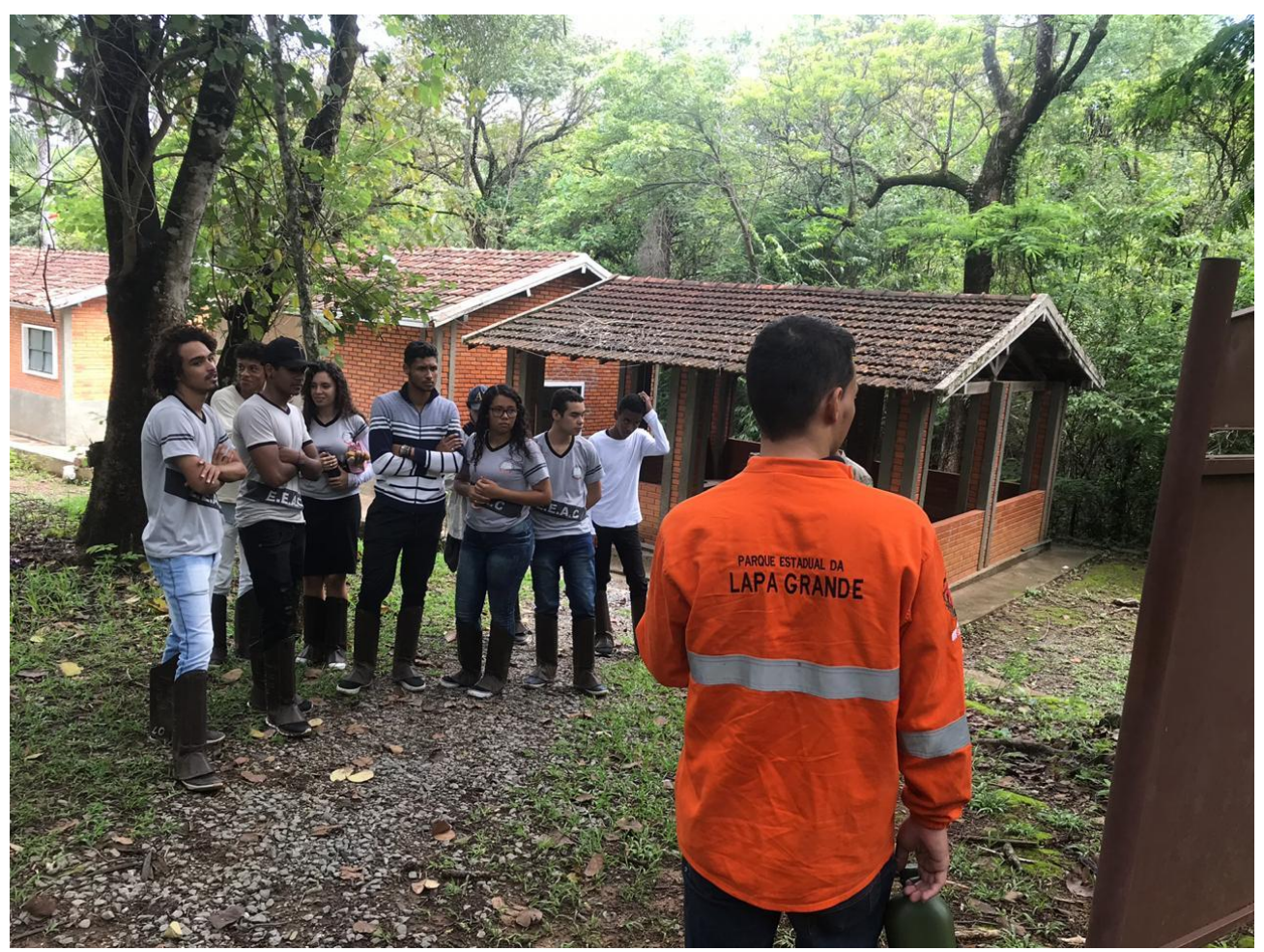

Fonte: Pedro Henrique Fonseca Veloso

Figura 3. Os alunos acompanhados da professora regente da disciplina de Biologia

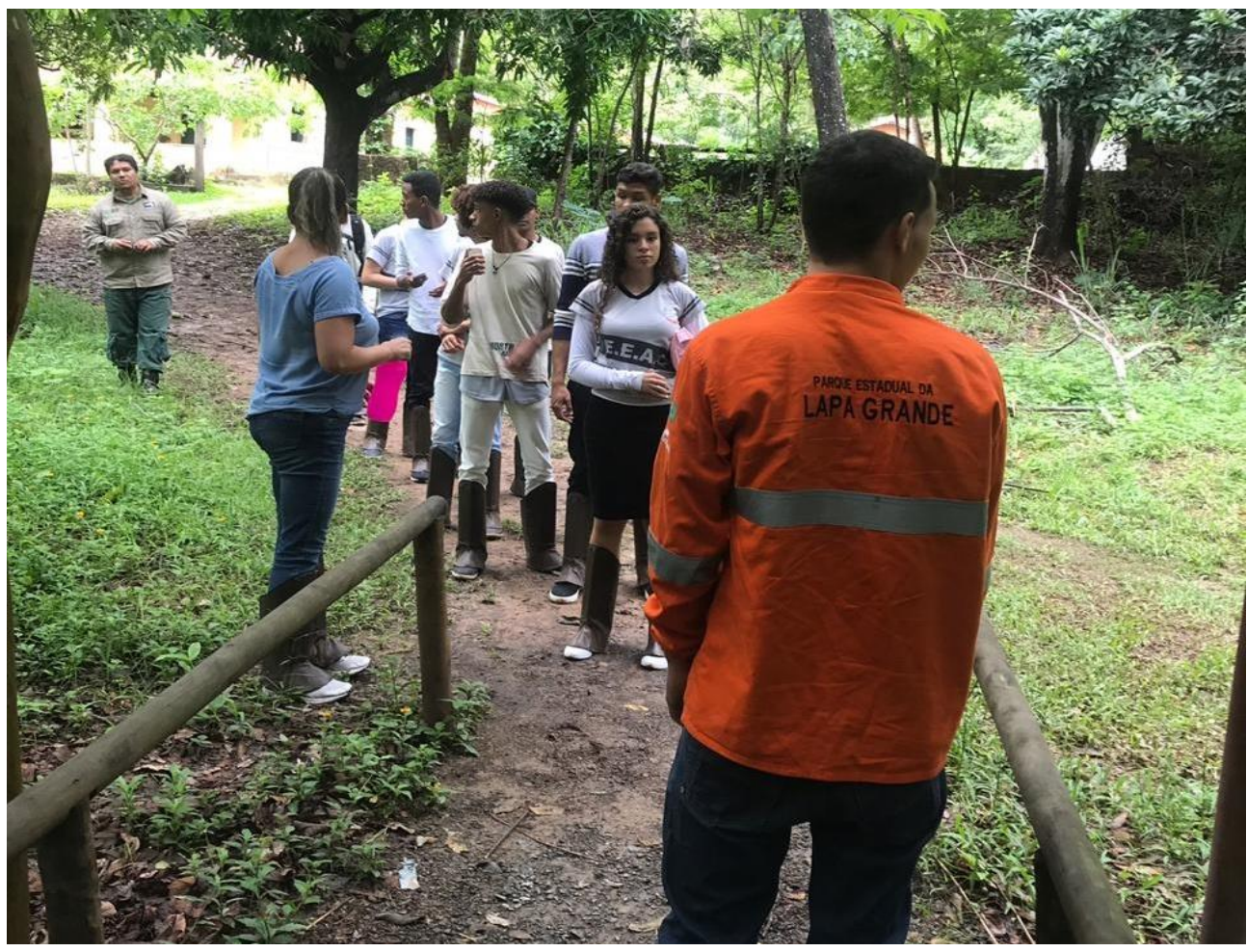


Meio Ambiente, botânica e solos: uma relação funcional para aulas de Biologia e Geografia Pedro Henrique Fonseca Veloso; Júlia Maria Viana Santana; Flávia Núbia Oliveira Miranda D’angelis; Ana Paula Venuto Moura; Guilherme Araújo Lacerda

Fonte: Pedro Henrique Fonseca Veloso

Figura 4. Os alunos e discente do curso de Ciências Biológicas Fazendo a trilha

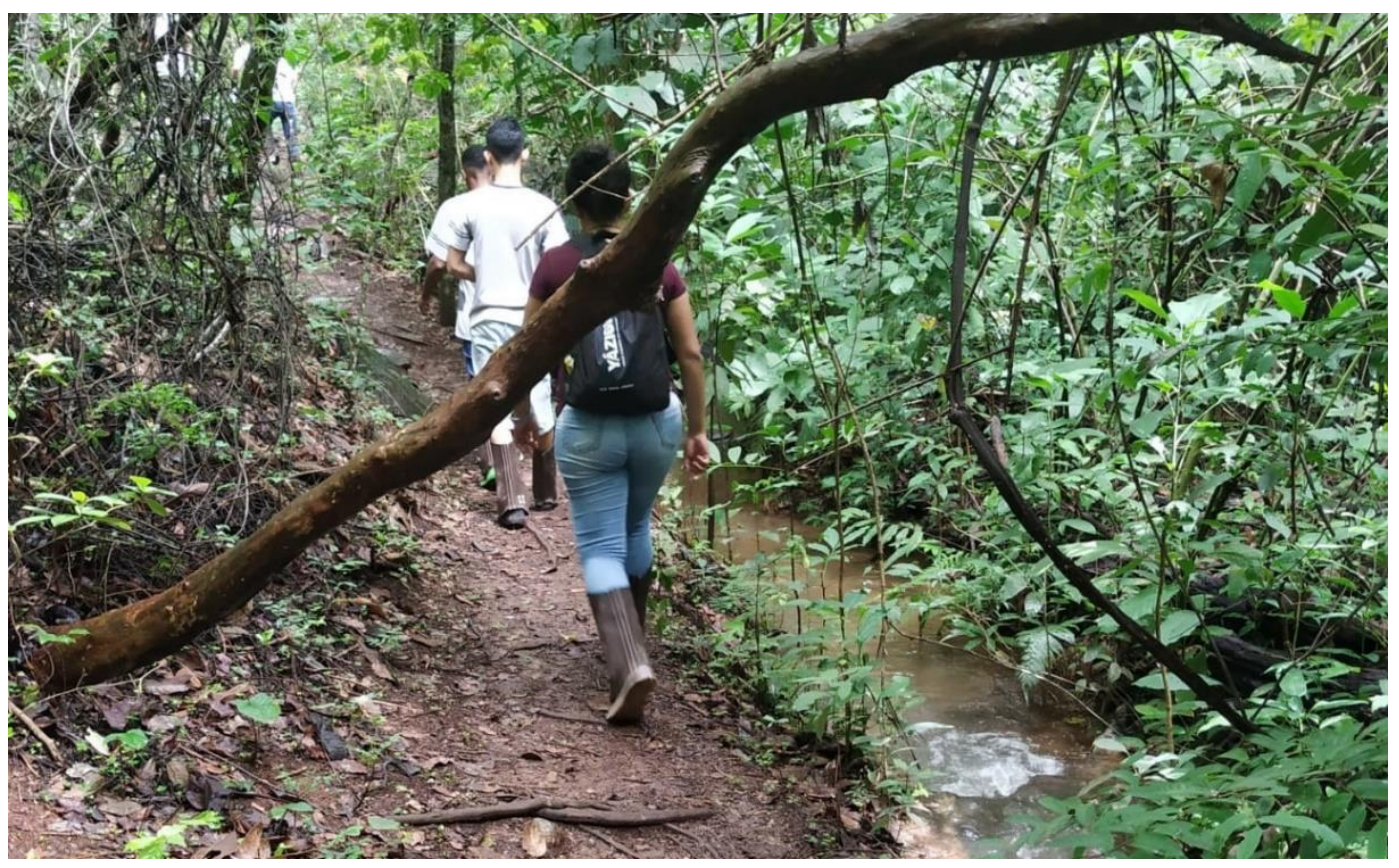

Fonte: Pedro Henrique Fonseca Veloso

No Brasil existe a LEI N 9.795, DE 27 DE ABRIL DE 1999 , denominada lei da Política Nacional de Educação Ambiental, que exige que as instituições de ensino tenha como pauta a educação ambiental em sua construção, como citado no " Art. $2^{\circ}$ A educação ambiental é um componente essencial e permanente da educação nacional, devendo estar presente, de forma articulada, em todos os níveis e modalidades do processo educativo, em caráter formal e não-formal” (BRASIL, 1999).

A aula fora da sala abre as portas para a realidade onde trabalhar com aspectos da fitofisionomia, solo e com o próprio ambiente, faz com que os alunos tenham a oportunidade de observar e questionar buscando obter respostas das suas principais dúvidas, onde pode ser observado nas figuras 5 e 6 . 
Meio Ambiente, botânica e solos: uma relação funcional para aulas de Biologia e Geografia Pedro Henrique Fonseca Veloso; Júlia Maria Viana Santana; Flávia Núbia Oliveira Miranda D'angelis; Ana Paula Venuto Moura; Guilherme Araújo Lacerda

Figura 5. Apresentação das características do parque

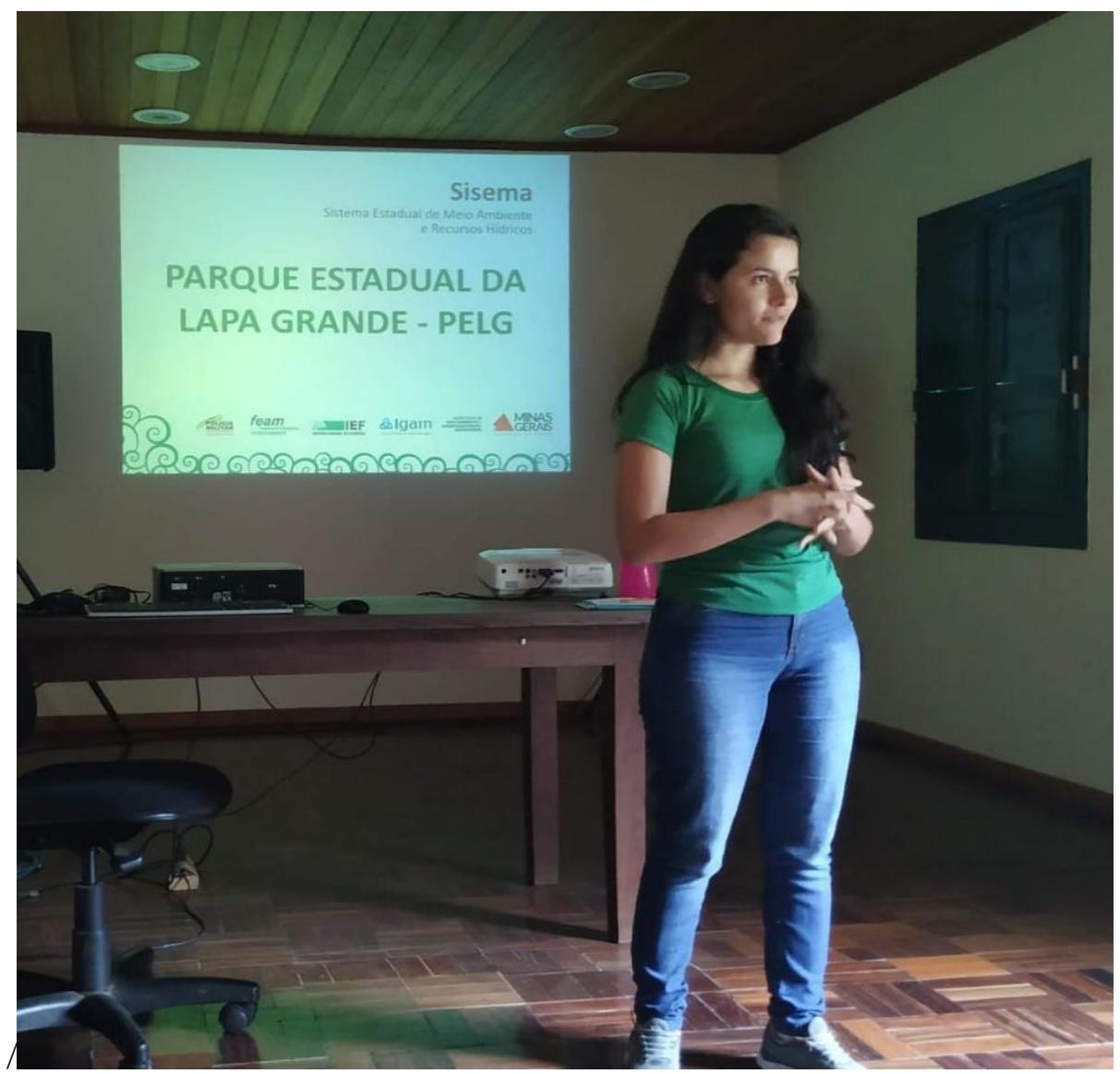

Fonte: Pedro Henrique Fonseca Veloso

Figura 6. Momento tira dúvidas

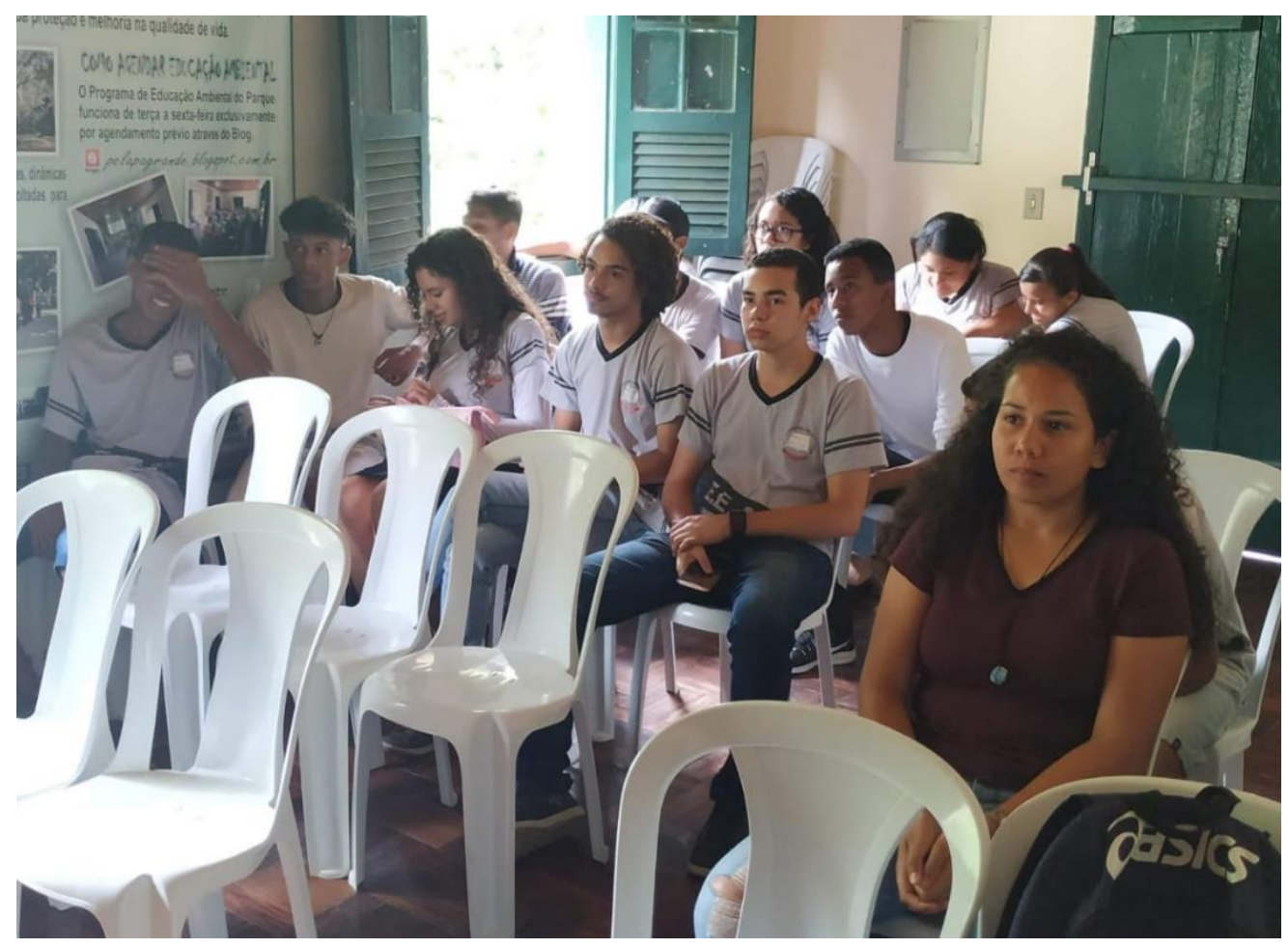

V.2, n.1, 2020 https://www.periodicos.unimontes.br/index.php/verdegrande 
Meio Ambiente, botânica e solos: uma relação funcional para aulas de Biologia e Geografia

Pedro Henrique Fonseca Veloso; Júlia Maria Viana Santana; Flávia Núbia Oliveira Miranda

D’angelis; Ana Paula Venuto Moura; Guilherme Araújo Lacerda

Fonte: Pedro Henrique Fonseca Veloso

O trabalho foi realizado durante o período do Programa Institucional de Bolsas de Iniciação à Docência (PIBID) em dezembro de 2019, com a finalidade de demonstrar a aula tida em sala de aula em uma aula prática demonstrativa no Parque Estadual da Lapa Grande, abordando diversas questões ambientais e a importância da conservação do bioma cerrado.

\section{Considerações finais}

Assim, nota-se a importância de aulas fora do ambiente escolar tanto para a socialização dos alunos fora do ambiente regular quanto para a proximidade dos mesmo com o meio ambiente externo, provocando assim uma melhor assimilação dos conteúdos ministrados em sala de aula.

Agradecemos a Coordenação de Aperfeiçoamento de Pessoal de Nível Superior (CAPES), pelo apoio financeiro ao Programa Institucional de Bolsas de Iniciação à Docência (PIBID) da qual fomos bolsistas.

\section{Referências}

BRASIL, Comissão de Políticas de Desenvolvimento. Lei n. 9.795, de 27 de abril de 1999: Dispõe sobre a educação ambiental, institui a Política Nacional de Educação Ambiental e dá outras providências. Diário Oficial da União, Brasília, v. 28, 1999. Disponível em: < http://www.planalto.gov.br/ccivil_03/leis/19795.htm > Acesso em: 8 mai. 2020.

DE MOURA CARVALHO, Isabel Cristina. Educação ambiental: a formação do sujeito ecológico. Ed. Cortez, 2017.

KLINK, Carlos A.; MACHADO, Ricardo B. A conservação do Cerrado brasileiro. Megadiversidade, v. 1, n. 1, p. 147-155, 2005.

MARACAHIPES, Leandro et al. Estrutura e composição florística da vegetação lenhosa em cerrado rupestre na transição Cerrado-Floresta Amazônica, Mato Grosso, Brasil. Biota Neotropica, v. 11, n. 1, p. 133-141, 2011. Disponível em: < https://www.scielo.br/scielo.php?pid=S1676-06032011000100013\&script=sci_arttext $>$

Acesso em: 5 mai. 2020.

MOREIRA, Adriana Aparecida et al. Análise do Comportamento Espectral de Fitofisionomias no Parque Estadual Lapa Grande por meio de dados MODIS. Revista Brasileira de Geografia Física, v. 6, n. 6, p. 0, 2013. Disponível em: < https://www.researchgate.net/profile/Fernando_Hiago_Souza_Fernandes/publication/2729063 22 Analysis_of the Spectral_Behavior_of_Physiognomies_in_Parque_Estadual_da_Lapa_G rande_by_means_of_MODIS_data/links/55f7047108aec948c46465a2/Analysis-of-the- 
Meio Ambiente, botânica e solos: uma relação funcional para aulas de Biologia e Geografia

Pedro Henrique Fonseca Veloso; Júlia Maria Viana Santana; Flávia Núbia Oliveira Miranda D’angelis; Ana Paula Venuto Moura; Guilherme Araújo Lacerda

Spectral-Behavior-of-Physiognomies-in-Parque-Estadual-da-Lapa-Grande-by-means-ofMODIS-data.pdf Acesso em: 3 mai. 2020.

NASCIMENTO-SILVA, Osmar; DE PAIVA, José Geraldo Antunes. Estudos Morfológicos e anatômicos em folhas adultas de Spondias tuberosa Arruda ( Anacardiaceae Lindley). Boletín latino-americano y del caribe de plantas medicinales y aromáticas, v. 6, n. 2, p. 36-43, 2007. Disponível em: < https://www.redalyc.org/pdf/856/85660206.pdf > Acesso em: 5 mai. 2020.

SCARIOT, Aldicir; SOUSA-SILVA, José Carlos; FELFILI, Jeanine Maria. Cerrado: ecologia, biodiversidade e conservação. Ministério do Meio Ambiente, 2005. Disponível em: < https://jbb.ibict.br/handle/1/361 > Acesso em: 4 mai. 2020. 